\title{
Development of a specific method to evaluate 8-hydroxy, 2-deoxyguanosine in sperm nuclei: relationship with semen quality in a cohort of 94 subjects
}

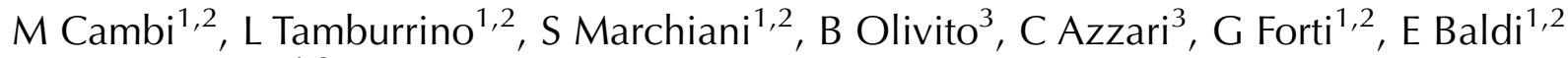 \\ and $M$ Muratori ${ }^{1,2}$ \\ ${ }^{1}$ Andrology Unit, Department of Clinical Physiopathology and ${ }^{2}$ Center of Excellence DeNothe, University of \\ Florence, Viale Pieraccini 6, I-50139 Florence, Italy and ${ }^{3}$ Department of Paediatrics, Anna Meyer Children Hospital, \\ Viale Pieraccini 24, 50132 Florence, Italy
}

Correspondence should be addressed to E Baldi and M Muratori at Andrology Unit, Department of Clinical Physiopathology, University of Florence; Email: elisabetta.baldi@unifi.it; monica.muratori@unifi.it

M Cambi and L Tamburrino contributed equally to this work

\begin{abstract}
Oxidative stress (OS) is involved in many disoders including male infertility. Human spermatozoa are very sensitive targets of reactive oxygen species (ROS) and most sperm functions are impaired in the case of OS. In addition unbalanced production of ROS is considered one of the most important causes of sperm DNA fragmentation, a semen trait of infertile men. The relationship between oxidative damage and semen quality is partially controversial, probably due to the different methods and/or targets used to reveal the OS. In this study, by fluorescence microscopy and flow cytometry, we compared two methods to reveal 8-hydroxy, 2-deoxyguanosine (8-OHdG), the hallmark of oxidative DNA damage: an immunofluorescence method and the commercial OxyDNA kit. We found that although both methods localized the labelling in sperm nuclei they yielded different measures, and only with the immunofluorescence method was the labelling specific for sperm 8-OHdG. The immunofluorescence method, coupled to flow cytometry, was thus selected to analyse the 8-OHdG content in semen samples from 94 subfertile patients and to investigate the relationship with semen quality. We found that the percentages of spermatozoa with 8-OHdG (mean \pm s.D., $11.4 \pm 6.9 \%$ ) were related to sperm count (Pearson's correlation coefficient $(r)=-0.27, P=0.04$ (ANOVA and student's $t$-test)), motility (progressive: $r=-0.22, P=0.04$; non-progressive: $r=0.25, P=0.01$ ), and normal morphology $(r=-0.27, P=0.01)$. In conclusion, we demonstrate that immunofluorescence/flow cytometry is a reliable and specific method to detect 8-OHdG at single-cell level and show that oxidative damage only partially overlaps poor semen quality, suggesting that it could provide additional information on male fertility with respect to routine semen analysis.

Reproduction (2013) $\mathbf{1 4 5} 227-235$
\end{abstract}

\section{Introduction}

Oxidative stress (OS) occurs when the production of free radicals and reactive oxygen species (ROS) overwhelms the activity of the antioxidant defences in the cell. This pathological condition has been involved in ageing and in many disorders, including male infertility (Tremellen 2008). In infertile subjects ROS levels increased and the antioxidant enzyme defences decrease with respect to donors or fertile men (Pasqualotto et al. 2001). Leukocytes appear to be the main source of ROS and thus infection/inflammation of male organs are considered aetiological factors of OS (Cocuzza et al. 2007), together with increased scrotal temperature due to fever, varicocele, cryptorchidism and exposure to toxicants (Lanzafame et al. 2009). In addition excessive
ROS production can occur directly in spermatozoa with abnormal head morphology and cytoplasmic retention (Gomez et al. 1996). Spermatozoa are believed to be very susceptible to OS, as they release most of the cytoplasm containing antioxidant enzymes during spermiogenesis (Aitken \& Curry 2011). In addition spermatozoa show a high content of polyunsaturated fatty acids in the membrane and such lipids are prone to peroxidation, a self-renewal process that provokes drastic effects on pivotal sperm functions. Indeed it has long been reported that lipid peroxidation negatively affects sperm motility, capacitation and competence for fertilizing the oocyte (Twigg et al. 1988). In addition OS has been proposed as an important, if not the main, cause of sperm DNA fragmentation (Aitken \& Curry 2011), which is found in a high percentage of ejaculated 
spermatozoa of sub- and infertile patients (Evenson et al. 1999, Aitken et al. 2010). Induction of sperm DNA breakage by OS may have important consequences, as the integrity of the sperm genome is a crucial trait for a safe and successful embryo development. In this scenario it has been proposed that the percentages of spermatozoa bearing signs of oxidative damage in the ejaculate might provide predictive information on male fertility status to add to the routine semen parameters (Tremellen 2008, Sakkas \& Alvarez 2010), poorly predictive of reproduction outcomes (Lewis 2007).

Overall, studies on the impact of OS on semen quality have reported controversial results. Sperm morphology results appeared to be negatively correlated with the level of OS in some studies (Shen et al. 1999, Chen et al. 2012) but not in others (Kao et al. 2008, Meseguer et al. 2008). According to the latter studies (Kao et al. 2008, Meseguer et al. 2008), the amount of oxidative damage affects sperm motility but neither sperm morphology nor sperm count. Furthermore a negative correlation between sperm count/density and OS was observed in some reports (Ni et al. 1997, Shen et al. 1999), however failing to reveal a relationship with motility (Shen et al. 1999) and motility and morphology ( $\mathrm{Ni}$ et al. 1997). Finally, some authors found no correlation between oxidative damage and semen quality (Thomson et al. 2009, Montjean et al. 2010, Zribi et al. 2011). These discrepancies may originate from the use of different techniques, and/or targets, to reveal OS in semen. Indeed, to investigate the role of OS in sperm biology and in general in male infertility many tools can be employed. These include evaluation of ROS production (Athayde et al. 2007) and/or the ability to counteract oxidative agents by enzyme and non-enzyme systems (Pasqualotto et al. 2008). In addition several studies investigated OS indirectly by revealing its noxious effects on sperm structure and function (Li et al. 2004), such as the formation of 8-hydroxy,2-deoxyguanosine $(8-\mathrm{OHdG})$, the hallmark of oxidative DNA damage. Among these studies, there are several investigations where 8-OHdG (Ni et al. 1997, Shen et al. 1999, Kao et al. 2008), is quantified using HPLC coupled to electrochemical detection (HPLC-EC). However, these methods are presently criticized as affected by the possible spontaneous formation of $8-\mathrm{OHdG}$ during the step of extraction/digestion of sperm DNA (Badouard et al. 2008). Recently, emerging techniques to reveal 8 -OHdG that employ antibodies (Kao et al. 2008) or binding proteins (De luliis et al. 2009, Zribi et al. 2010) have been reported.

In this study, we compared two methods to detect sperm 8-OHdG at the single-cell level: the OxyDNA kit, based on a binding protein to $8-\mathrm{OHdG}$, and an immunofluorescence procedure employing a $\mathrm{MAB}$ against 8-OHdG moiety. As only the latter was capable of specifically assessing the amount of oxidative DNA damage in spermatozoa, we coupled the immunofluorescence technique to flow cytometry detection to analyse the percentage of 8-OHdG-positive spermatozoa from 94 subfertile patients and its relationship with semen quality.

\section{Results}

\section{Comparing two methods for 8-OHdG detection}

In spermatozoa stained by propidium iodide (PI), we revealed oxidative DNA damage by both the BP-F provided in the oxyDNA kit and the MAB 15A3 against $8-\mathrm{OHdG}$, the latter in turn revealed with a fluorescent secondary antibody. After examination of spermatozoa by fluorescence microscope in order to localize the green fluorescence signals due to the antibody (Fig. 1A) and the BP-F (Fig. 1B), we observed that, as expected, the labelling occurred in the sperm nuclei with both the techniques (Fig. 1). When fluorescence was revealed by flow cytometry, we found that the percentages of 8-OHdG-labelled spermatozoa revealed with the oxyDNA kit were much greater than those obtained with the immunofluorescence method. On average, the
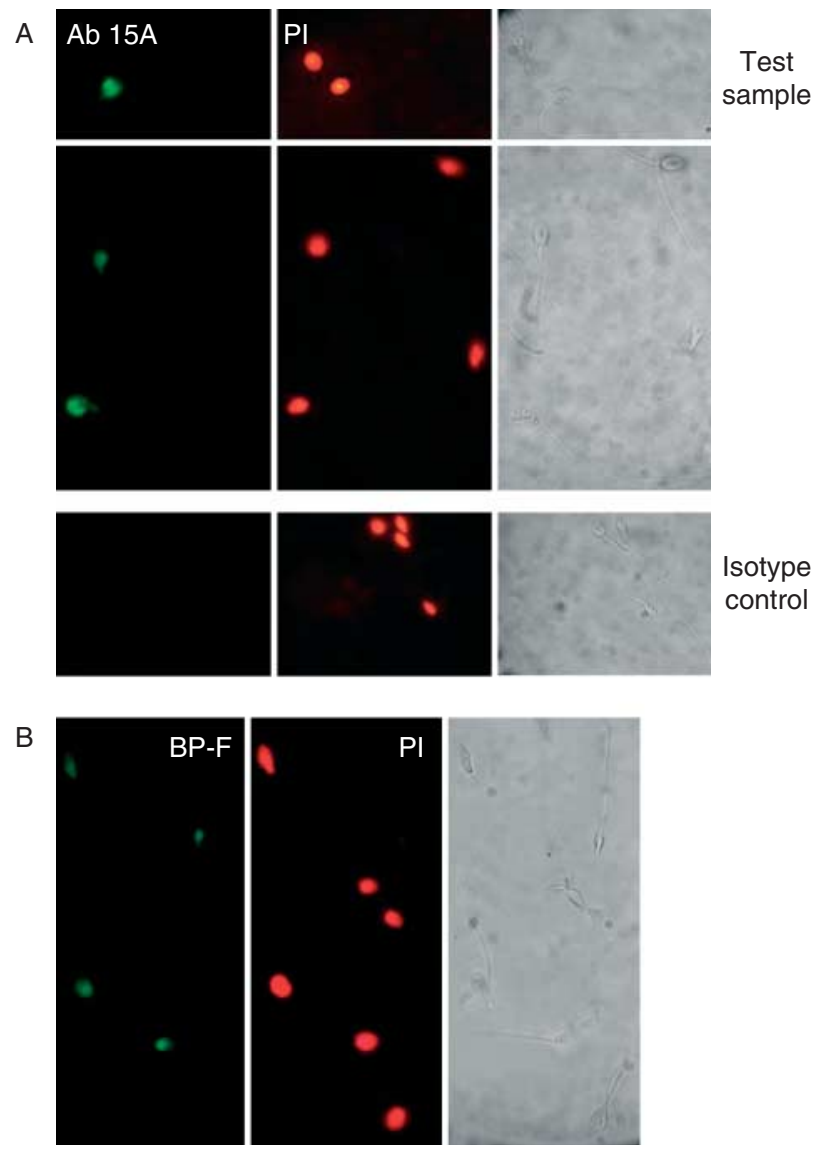

Figure 1 Micrographs of sperm samples double stained for nuclei $(\mathrm{PI})$ and $8-\mathrm{OHdG}$, the latter with an immunofluorescence procedure (A) or with OxyDNA kit (B). For the immunofluorescence method, images from the sample incubated with the isotype control IgG2a are shown in the lower panels $(\mathrm{A})$. 

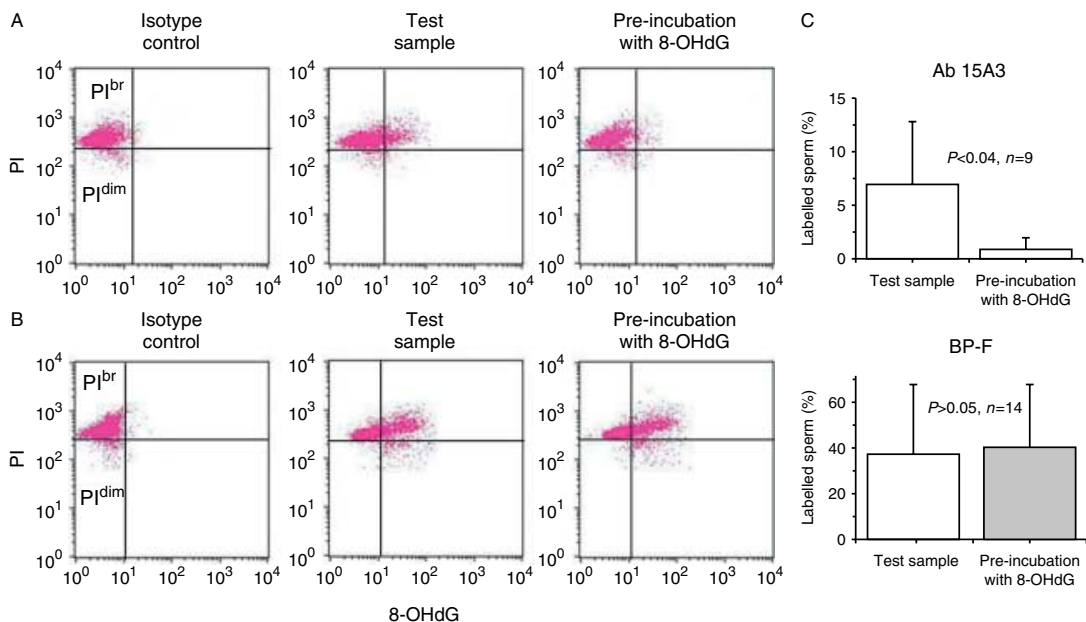

Figure 2 Typical dot plots showing fluorescence of $\mathrm{PI}$ and $8-\mathrm{OHdG}$, the latter obtained with an immunofluorescence procedure (A) and with the OxyDNA kit (B). Note that PI staining unveils two different sperm populations indicated as $\mathrm{Pl}^{\mathrm{br}}$ and $\mathrm{Pl}^{\mathrm{dim}}$ (Muratori et al. 2008, Marchiani et al. 2011, Meamar et al. 2012). Effect of exceeding concentrations of $8-\mathrm{OHdG}$ on the antibody $15 \mathrm{~A} 3$ (A, right panels) and the BP-F (B, right panels) ability to detect $8-\mathrm{OHdG}$. Results from one typical experiment ( $A$ and $B$ ) and average values from the indicated numbers of experiments $(C)$ are shown. amount of oxidative DNA damage was $35.5 \pm 28.6 \%$ $(n=30)$ with the oxyDNA kit and $11.4 \pm 6.9 \%(n=94$, $P<0.05$ vs OxyDNA kit) with the immunofluorescence method. Figure 2 shows typical dot plots of the same semen sample stained for $8-\mathrm{OHdG}$ detection with the immunofluorescence method (Fig. 2A, left and medium panels) and oxyDNA kit (Fig. 2B, left and medium panels). As can be observed, in case of the OxyDNA kit, a shift to the right of the entire sperm population in the test sample with respect to the negative control is present, suggesting a binding of BP-F to all cells. A marked shift of BP-F labelling in spermatozoa has been reported also by other authors (Banihani et al. 2012). The lower amount of 8-OHdG with the immunofluorescence procedure could be due to a lower access of the antibody into the sperm nuclei with respect to the BP-F of the kit. However, no difference in sperm labelling was found when the immunofluorescence procedure was performed after a step of decondensation of chromatin with dithiothreitol (DTT; $2 \mathrm{mM}, 45 \mathrm{~min}$ at $37^{\circ} \mathrm{C}$, data not shown).

To assess the specificity of the two methods, we investigated the ability of the nucleoside $8-\mathrm{OHdG}$ to prevent the binding of the antibody and of the BP-F to sperm nuclei. We found that pre-incubation of the antibody with 8 -OHdG $(\sim 1: 1000)$ almost completely blunted the green fluorescence signal of sperm nuclei $(6.9 \pm 5.8$ vs $0.9 \pm 1.0, n=8$, Fig. $2 \mathrm{~A}$ right panel). In contrast, similar pre-incubation of BP-F with the standard nucleoside had no effect on the fluorescence obtained with the oxyDNA kit (37.2 \pm 30.7 vs $40.4 \pm 27.7, n=11$; Fig. $2 B$ right panel), even when BSA was omitted to avoid a possible withdrawal of the nucleoside standard from the medium of incubation with BP-F (results not shown). Figure $2 \mathrm{C}$ shows the average percentages of labelled spermatozoa obtained with or without pre-incubation with the excess of 8-OHdG of the antibody (upper columns) or the BP-F (lower columns).
To further validate the immunofluorescence method, we verified whether it could detect the expected increase in the $8-\mathrm{OHdG}$ amount provoked by an oxidative insult. To this aim, we incubated sperm with $\mathrm{H}_{2} \mathrm{O}_{2}(25 \mu \mathrm{M}$, for $1 \mathrm{~h}$ at $37^{\circ} \mathrm{C}, n=11$ ) and found that the treatment was able to decrease the progressive motility (with vs without $\mathrm{H}_{2} \mathrm{O}_{2}: 11.6 \pm 13.8$ vs $\left.23.7 \pm 18.0, P<0.01\right)$ without significantly affecting the percentage of immotile sperm (with vs without $\mathrm{H}_{2} \mathrm{O}_{2}: 52.5 \pm 30.7$ vs $42.4 \pm 19.4$, $P>0.05)$ and thus the sperm viability as previously shown by our group (Muratori et al. 2003). However, the treatment was able to increase the amount of sperm $8-\mathrm{OHdG}$ (percentage increase with respect to the basal level: $59.5 \pm 44.8 \%, P<0.005, n=11$ ). Figure 3 reports representative dot plots of a labelled semen sample treated (right panel) or not (medium panel) with $\mathrm{H}_{2} \mathrm{O}_{2}$.

\section{Relationship between 8-OHdG levels and semen quality}

In light of the results obtained from the comparison between the OxyDNA kit and the immunofluorescence method, we selected the latter to determine the percentage of sperm with oxidative DNA damage in 94 subfertile patients, where we found an average value of $11.4 \pm 6.9 \%$ (range, $1.9-32.1 \%$ ). The occurrence of $8-\mathrm{OHdG}$ was detected in spermatozoa counterstained with PI (Figs 1, 2 and 3) in order to eliminate anucleated interferents present in semen (Marchiani et al. 2007, Muratori et al. 2008). Besides allowing a more accurate evaluation of sperm parameters (Muratori et al. 2008, Marchiani et al. 2011), PI staining reveals the occurrence of two different sperm populations, named $\mathrm{Pl}^{\mathrm{br}}$ and $\mathrm{PI}^{\mathrm{dim}}$, as recently reported by our group (Muratori et al. 2008, Marchiani et al. 2011, Meamar et al. 2012). Interestingly, in all tested samples, only $\mathrm{Pl}^{\mathrm{br}}$ spermatozoa showed signs of oxidative DNA damage, whereas virtually no cell in $\mathrm{Pl}^{\text {dim }}$ population presented $8-\mathrm{OHdG}$ residues (Figs 2 and 3). 


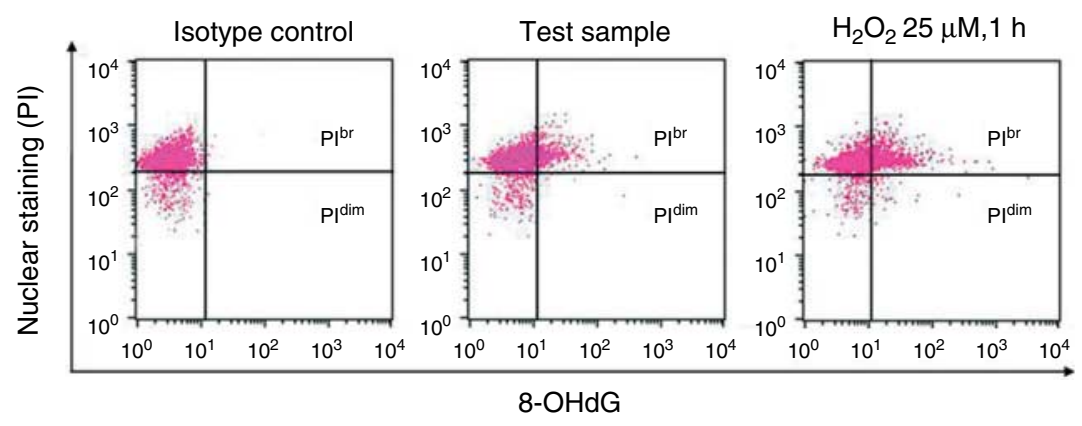

Figure 3 Effect of sperm treatment with $\mathrm{H}_{2} \mathrm{O}_{2}$, $25 \mu \mathrm{M}\left(1 \mathrm{~h}, 37^{\circ} \mathrm{C}\right)$ on the 8 -OHdG labelling as assessed using the antibody $15 \mathrm{~A} 3$ and revealed in PI-stained sperm. A typical flow cytometric result out of 11. Note that PI staining unveils two different sperm populations indicated as $\mathrm{PI}^{\mathrm{br}}$ and $\mathrm{PI}^{\mathrm{dim}}$ (Muratori et al. 2008, Marchiani et al. 2011, Meamar et al. 2012) and that only $\mathrm{Pl}^{\mathrm{br}}$ spermatozoa show 8-OHdG fluorescence.
To investigate the relationship between sperm oxidative DNA damage and semen quality, we correlated the amounts of oxidative DNA damage with standard semen parameters of the recruited patients. Table 1 reports the Pearson correlation coefficients and the corresponding $P$ values between the percentages of damaged spermatozoa and semen parameters, showing that sperm DNA oxidative damage is associated with poor semen quality. Indeed, the levels of $8-\mathrm{OHdG}$ negatively correlated with progressive motility, morphology and count of spermatozoa and positively with non-progressive motility (Fig. 4, Table 1). No correlation was found with sperm concentration, ejaculate volume, $\mathrm{pH}$ and abstinence length (Table 1). The $8-\mathrm{OHdG}$ amount detected in the non-smokers $(11.6 \pm 6.8, n=66)$ did not differ $(P>0.05)$ from that of the smokers (11.4 $\pm 7.2, n=28)$. Moreover, among smokers, no difference was observed between light $(<10$ cigarettes/day, $10.5 \pm 7.8, n=16)$ and heavy ( $>10$ cigarettes/day, $12.5 \pm 6.4, n=12$ ) smokers.

To further investigate the relationship between semen quality and the amount of oxidative sperm DNA damage, we evaluated 8-OHdG in sperm fractions of differing quality, prepared by a 40 and $80 \%$ discontinuous PS100 centrifugation gradient (Aitken et al. 1993). As $8-\mathrm{OHdG}$ was found only in $\mathrm{Pl}^{\text {br }}$ sperm (see above), the different incidence of $\mathrm{Pl}^{\text {dim }}$ spermatozoa in the two fractions (Muratori et al. 2008) might represent a confounding variable in the calculation of the percentage of $8-\mathrm{OHdG}$ in the two fractions. Hence, in these experiments, the percentage of cells with oxidative DNA damage was calculated only in the $\mathrm{Pl}^{\mathrm{br}}$ population. With respect to the $40 / 80 \%$ fraction, the percentage of sperm with oxidative damage in the $80 \%$ fraction was lower in six out of eight subjects and increased or unchanged in the remaining two (Fig. 5). These results confirm that sperm oxidative DNA damage is only weakly associated with standard semen parameter and the association can be revealed only after analysis of a large number of subjects $(n=94)$.

\section{Discussion}

In this study we assessed the amount of sperm 8-OHdG at single-cell level in subfertile men and investigated its relationship with semen quality. We found that all the standard semen parameters correlated, although weakly, with the percentages of spermatozoa with $8-\mathrm{OHdG}$. A weak correlation between nuclear oxidative status and semen quality suggests that oxidative damage may be found in semen with normal motility, morphology and count as assessed by routine semen analysis. Hence, the assessment of the amount of sperm oxidative damage could provide additional information on male fertility status with respect to the poorly predictive standard semen parameters (Lewis 2007).

The method used in this study to reveal sperm 8-OHdG was selected after comparison of an immunofluorescence method set up in our laboratory with a very popular procedure to detect oxidative DNA damage in individual sperm, the commercial oxyDNA kit. The OxyDNA kit is based on the use of a fluorescent binding protein that localizes the yielded fluorescence in sperm nuclei (Santiso et al. (2010) and this study). In our hands, OxyDNA kit produced measures $(37.2 \pm 30.7 \%)$ comparable to those previously reported in similar sperm preparations (Meseguer et al. 2008, Aitken et al. 2010, Bellver et al. 2010), albeit, on average, fourfold greater than those yielded by the immunofluorescence method $(11.4 \pm 6.9 \%)$. It is anticipated that such a discrepancy is not due to a hampered access of the antibody molecules into sperm nuclei as no difference was observed when the $8-\mathrm{OHdG}$ labelling was obtained after a step of

Table 1 Pearson's correlation between the percentages of sperm with $8-\mathrm{OHdG}$ and the main semen parameters, age and abstinence.

\begin{tabular}{lcccc}
\hline & & & \multicolumn{2}{c}{ 8-OHdG } \\
\cline { 5 - 5 } Parameters & Mean \pm s.D. & $\boldsymbol{n}$ & $r$ & $P$ \\
\hline $\begin{array}{l}\text { Sperm count } \\
\quad \text { (sperm/ejaculate) }\end{array}$ & $235.5 \pm 192.2$ & 94 & -0.27 & $\leq 0.04$ \\
$\begin{array}{l}\text { Sperm concentration } \\
\quad \text { (sperm/ml) }\end{array}$ & $77.9 \pm 58.2$ & 94 & -0.18 & $\leq 0.09$ \\
$\begin{array}{l}\text { Progressive motility (\%) } \\
\text { Non-progressive }\end{array}$ & $49.9 \pm 16.0$ & 94 & -0.22 & $\leq 0.04$ \\
$\quad 10.5 \pm 4.7$ & 94 & 0.25 & $\leq 0.01$ \\
$\quad$ motility (\%) & & & & \\
Immotile sperm (\%) & $40.1 \pm 14.6$ & 94 & 0.16 & $\leq 0.13$ \\
Normal morphology (\%) & $8.8 \pm 6.1$ & 94 & -0.27 & $\leq 0.01$ \\
Volume (ml) & $3.3 \pm 1.3$ & & -0.12 & $\leq 0.23$ \\
pH & $7.5 \pm 0.2$ & & -0.04 & $\leq 0.66$ \\
Age (years) & $34.1 \pm 6.9$ & & -0.2 & $\leq 0.10$ \\
Abstinence (days) & $4.0 \pm 1.8$ & & 0.12 & $\leq 0.23$ \\
\hline
\end{tabular}



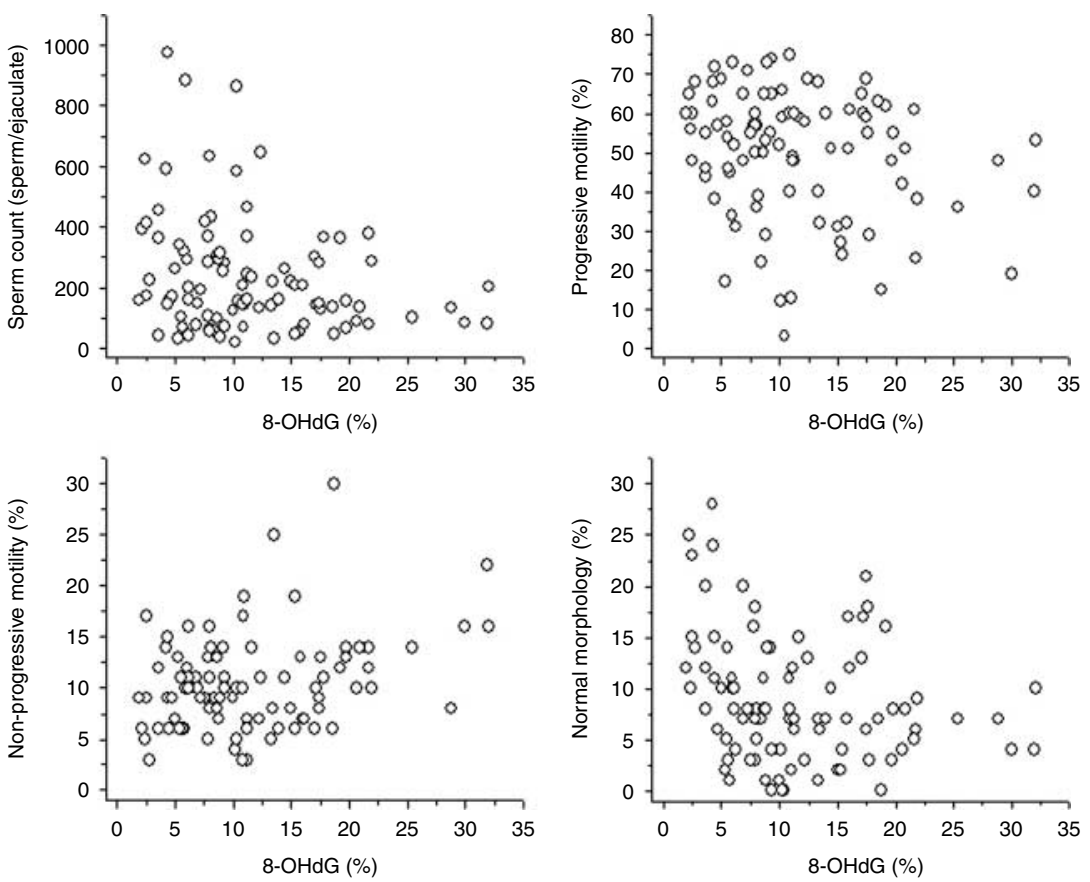

Figure 4 Relationship between the $8-\mathrm{OHdC}$ amounts in sperm and standard semen parameters. Scatter plots between the percentages of sperm with 8-OHdG residues from 94 semen samples, and sperm count, progressive and nonprogressive motility and normal morphology. chromatin decondensation. Furthermore we previously demonstrated (Marchiani et al. 2011) that the immunolabelling of sperm nuclear proteins is almost complete if a protocol employing a $1 \mathrm{~h}$ permeabilization step, similar to the method used in this study, is applied. Both methods are able to detect the expected increase in $8-\mathrm{OHdG}$ content induced by an oxidative insult that was a treatment with $4 \mathrm{M} \mathrm{H}_{2} \mathrm{O}_{2}$ (Zribi et al. 2011) or Fenton reaction (De luliis et al. 2009) for the oxyDNA kit and incubation with $25 \mu \mathrm{M} \mathrm{H}_{2} \mathrm{O}_{2}$ for the immunofluorescence method (this study). In our hands, $25 \mu \mathrm{M} \mathrm{H}_{2} \mathrm{O}_{2}$ is able to induce massive damage to sperm membrane (Muratori et al. 2003) as well as a reduction of progressive motility (this study) without increasing the amount of sperm with severe cell damage that could undergo degradation during the treatment and thus provoke a cell selection (Muratori et al. 2010). Even using a relatively low dose of $\mathrm{H}_{2} \mathrm{O}_{2}$, the oxidative DNA damage is produced and the immunofluorescence method is able to detect it, thus resulting very sensitive.

To further compare the two methods, and to assess the specificity of the probes used to reveal $8-\mathrm{OHdG}$ in the nuclei, competitive experiments were performed with both the 15A3 antibody and the BP-F. We found that in the presence of an excess of $8-\mathrm{OHdG}$ sperm labelling with BP-F was unaffected whereas under the same conditions the fluorescent signal due to the antibody was completely blunted. The latter finding suggests that whereas the binding of the antibody to sperm nuclei for the oxidated nucleoside is specific, that of the BP-F is not. To our knowledge, none of the studies assessing 8-OHdG in spermatozoa with the OxyDNA kit reported results of competitive experiments with the
BP-F. After a careful survey of the literature, we came to the conclusion that the basis for the development of the oxyDNA kit comes from an investigation (Struthers et al. 1998) on the binding between 8-OHdG and avidin, a tetrameric protein found in the egg white and extensively used in binding assays for its high affinity for the vitamin biotin. In a later report of Conners et al. (2006), avidin, itself or a later engineered form, is indeed indicated as

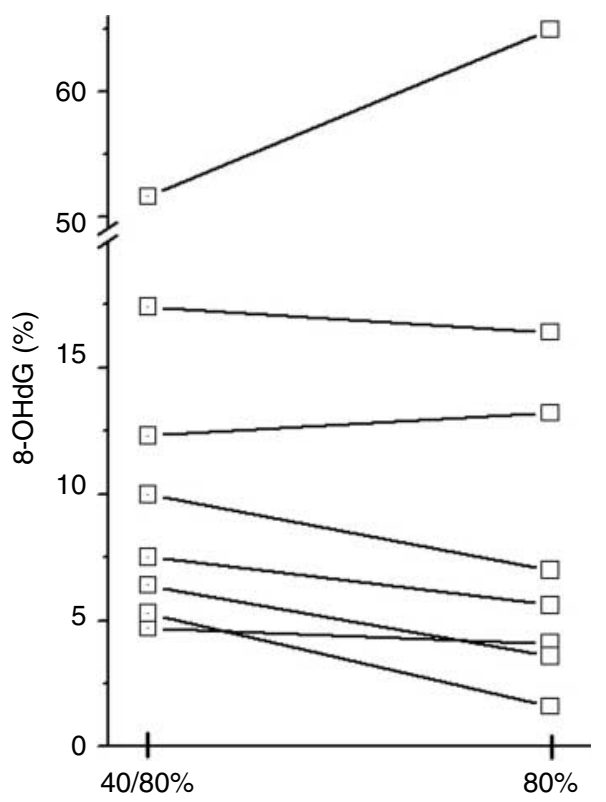

Figure 5 8-OHdG amounts in the two sperm fractions recovered by density gradient centrifugation in eight semen samples. Percentages of 8-OHdG-positive spermatozoa were calculated in the $\mathrm{Pl}^{\text {br }}$ population (see text). 
the BP-F of the commercial kits for the detection of 8-OHdG. Avidin binds DNA at high affinity and forms with it stable and organized complexes (Morpurgo et al. 2004). In these complexes, the binding between avidin and DNA is supposed (Morpurgo et al. 2004) to be mainly due to a predominance of positively charged residues on the surface of avidin, thus to non-specific electrostatic interactions (Conners et al. 2006, Takakura et al. 2012). Conversely, Struthers et al. (1998) showed that the co-incubation of avidin with 8-OHdG inhibited the binding with oxidized fragments of DNA and with cultured cells treated with $\mathrm{H}_{2} \mathrm{O}_{2}$, even if such an inhibition was only partial (about $60 \%$ in case of the DNA fragments), suggesting both the specificity of a certain amount of the binding for $8-\mathrm{OHdG}$ and the persistence of unspecific interactions. Although we cannot exclude that, in the current commercial kits, avidin has been engineered to reduce the non-specific binding (Takakura et al. 2012), in our hands, the co-incubation of BP-F with $8-\mathrm{OHdG}$ did not result in a decrease of the fluorescence signal to sperm nuclei. It is possible that, at variance with DNA macromolecules and cultured somatic cells (employed in the study by Struthers et al. (1998)), in the highly compacted sperm chromatin, the specific binding between BP-F and $8-\mathrm{OHdG}$, if any, is masked by the fluorescence due to non-specific interactions and/or that it can be unmasked only when chromatin is heavily oxidized as in the cases of treatment with huge concentrations of $\mathrm{H}_{2} \mathrm{O}_{2}$ (Zribi et al. 2011) or Fenton reaction induction (De luliis et al. 2009). In line with this conclusion is the finding (Zribi et al. 2011) that the amount of 8-OHdG assessed by the oxyDNA kit does not associate with that of another marker of OS, i.e. the malondialdehyde in the semen of a group of patients similar to this study. Intriguingly, in the study by Zribi et al. (2011), sperm levels of spermatozoa were associated with poor sperm motility whereas those of $8-\mathrm{OHdG}$ were not.

In our study, the labelling of $8-\mathrm{OHdG}$ was coupled to flow cytometry detection that allowed us to reveal 8-OHdG separately in the $\mathrm{PI}^{\text {br }}$ and $\mathrm{PI}^{\text {dim }}$ sperm populations, showing a variable incidence among subfertile subjects (Muratori et al. 2008, Marchiani et al. 2011, Meamar et al. 2012). These populations occur in human semen and were revealed because of a difference in PI staining (Muratori et al. 2008), detectable in flow cytometry but not in fluorescence microscopy. $\mathrm{Pl}^{\mathrm{br}}$ and $\mathrm{Pl}^{\text {dim }}$ sperm populations differ in the extent of DNA fragmentation and the relationship between DNA breakage and semen quality (Muratori et al. 2008). Indeed, at variance with the $\mathrm{Pl}^{\text {br }}$ population, the $\mathrm{PI}^{\text {dim }}$ spermatozoa are DNA fragmented, representing the sperm population that drives the relationship between sperm DNA fragmentation and poor semen quality (Muratori et al. 2008). In addition, by labelling dead sperm in fresh samples with L23101 stain, that remains after the procedure to reveal the two sperm populations, we found that $\mathrm{PI}^{\mathrm{dim}}$ spermatozoa are unviable, whereas in $\mathrm{Pl}^{\mathrm{br}}$ population, there are both live and dead cells (Marchiani et al. 2011). In this study, we found that 8-OHdG is present only in $\mathrm{Pl}^{\text {br }}$ population (see Figs 2 and 3), whereas $\mathrm{Pl}^{\text {dim }}$ spermatozoa did not show this sign of oxidative damage, in any of the recruited subjects. The finding that oxidative damage is not present in $\mathrm{Pl}^{\text {dim }}$ spermatozoa, which are unviable, is consistent with the assumption that production of ROS depends on the aerobic metabolism of live cells. The lack of labelling for 8-OHdG in DNA fragmented $\mathrm{PI}^{\text {dim }}$ spermatozoa indicates that DNA breakage is not concomitant with oxidative DNA damage and that, at least in this population, DNA fragmentation might originate by mechanisms other than a direct attack of ROS to sperm chromatin (Aitken \& Curry 2011), such as apoptosis or impairment during sperm chromatin packaging (Muratori et al. 2006, Tamburrino et al. 2012). The lack of correlation between the levels of malondyaldeide and those of sperm DNA breakage recently reported by Montjean et al. (2010) appears also consistent with this conclusion. However, we cannot completely exclude a role of oxidative attack in generating DNA breaks in the non-viable $\mathrm{Pl}^{\text {dim }}$ spermatozoa that might represent a late step of a degenerative process triggered by $\mathrm{OS}$ and terminated in extensive DNA fragmentation and loss of oxidative adducts.

In our study, we found an average percentage value of 8-OHdG-positive spermatozoa of $11.4 \pm 6.9 \%$, in 94 male partners of infertile couples. It is possible that such a value does not exactly reflect the real amount of sperm oxidative DNA damage in infertile men, as our recruited patient population could include fertile partners of infertile women.

In line with previous studies ( $\mathrm{Ni}$ et al. 1997, Shen et al. 1999, Kao et al. 2008), we found a weak, albeit significant, negative association between oxidative DNA damage and semen quality. However, these studies employed a HPLC-EC method that may result in overestimation of the parameter, due to the possible occurrence of spontaneous oxidation of DNA (Badouard et al. 2008). The negative effect of oxidative insult on sperm count (Ni et al. (1997) and Shen et al. (1999), this study) and motility (Shen et al. (1999) and Kao et al. (2008), this study) is not surprising, as viability and motility are sperm features critically dependent on the integrity of the membrane that, in turn, is a very sensitive target of OS. The study of Shen et al. (1999) also reports a positive correlation between $8-\mathrm{OHdG}$ amounts and the percentages of both abnormal morphology of sperm, as in this study, and head anomalies. A correlation between oxidative damage to DNA and sperm morphology might be the result of the occurrence of abnormal spermatozoa due to an incomplete maturation, which, in turn, may produce ROS because of excessive cytoplasm retention (Gomez et al. 1996). On the other hand, sperm anomalies deriving from 
a putative impairment of maturation and, in particular of chromatin packaging, would render spermatozoa more susceptible to ROS attack on DNA nucleotides. Overall the present investigation is consistent with the studies (Ni et al. 1997, Shen et al. 1999, Chen et al. 2012) indicating that the association between OS and global semen quality is weak or even absent and that, similar to what has been reported for sperm DNA fragmentation (Cohen-Bacrie et al. 2009), the detection of oxidative damage in semen could add diagnostic information to the routine semen analysis in the evaluation of male infertility (Tremellen 2008, Sakkas \& Alvarez 2010).

In conclusion, the use of a reliable and specific method to detect $8-\mathrm{OHdG}$ at the single-cell level demonstrates a weak negative association between this sign of oxidative damage and all standard semen parameters, supporting the idea that the determination of OS in semen can improve the diagnostic value of semen analysis.

\section{Materials and Methods}

\section{Chemicals}

Human tubal fluid (HTF) medium and human serum albumin (HSA) were purchased from Celbio (Milan, Italy). Diff-Quick kit was purchased from CGA, Diasint (Florence, Italy). BSA was purchased from ICN Biomedicals (Irvine, California, USA). The monoclonal mouse antibody anti-8-OHdG, 15A3, was from Santa Cruz Biotechnology and mouse IgG2a isotype control antibody was purchased from Exbio (Praha, Czech Republic). 8-OHdG was purchased from both BioLogLife Science Institute (Bremen, Germany) and Zeptometrix Corporation (Buffalo, NY, USA). Biotrin OxyDNA test was from Argutus Medical (Dublin, Ireland). Paraformaldehyde (PFA) was obtained from Merck Chemicals. The other chemicals were from Sigma Chemical.

\section{Ethics statement}

The study has been approved by the Local Ethics Committee of the Azienda Ospedaliera e Universitaria (AOUC) Careggi, and informed written consent has been obtained from the recruited patients.

\section{Semen samples collection, preparation and treatment}

Semen samples were consecutively collected, according to WHO criteria (World Health Organization 1999), from men undergoing routine semen analysis as part of testing of couples with fertility problems in the Andrology Laboratory of the University of Florence. Subjects undergoing drug therapies were excluded from the study as well as semen samples with detectable leucocytes. Conversely, subjects with a smoking habit (light and heavy smokers: less and more than ten cigarettes/day respectively) were included in the study. Sperm morphology and motility were assessed by optical microscopy according to WHO criteria (World Health Organization 1999). Sperm morphology was evaluated by determining the percentage of normal and abnormal forms after Diff-Quik staining, by scoring at least 100 spermatozoa/slide. Sperm motility was scored by determining the percentage of progressively motile, non-progressively motile and immotile spermatozoa by scoring of at least 100 spermatozoa/slide. The tests on the neat semen $\left(10^{7}\right.$ spermatozoa/sample) were performed after washing twice with HTF medium and fixation with PFA $(500 \mu \mathrm{l}, 4 \%$ in PBS, $\mathrm{pH} 7.4)$ for $30 \mathrm{~min}$ at room temperature. Treatment of spermatozoa with hydrogen peroxide was conducted after washing twice with HTF medium and by incubating at $25 \mu \mathrm{M}$ for $1 \mathrm{~h}$ at $37^{\circ} \mathrm{C}$ in the same medium.

Sperm selection by density gradient centrifugation was achieved using a $40 \%$ and $80 \%$ discontinuous Pure Sperm 100 (PS100, Nidacon, Mölndal, Sweden) gradient. For this procedure, PS100 was diluted in HTF medium containing $10 \%$ HSA (HTF-HSA) and up to $2 \mathrm{ml}$ semen were layered on top of each gradient and centrifuged at $500 \mathrm{~g}$ for $30 \mathrm{~min}$. Following centrifugation, the seminal plasma and PS100 were discarded and spermatozoa were recovered from the pellet of the $80 \%$ PS100 fraction (80\% fraction) and from the interface of the $40 \%$ and $80 \%$ (40/80\% fraction) gradients. Finally, spermatozoa were washed twice in HTF-HSA and fixed $\left(10^{7}\right.$ cells $)$ as described earlier.

\section{Detection of 8-OHdG by immunofluorescence method}

For detection of 8-OHdG, fixed sperm samples were washed twice with $1 \%$ NGS-PBS and split into two aliquots subsequently incubated $\left(1 \mathrm{~h}\right.$ at $\left.37^{\circ} \mathrm{C}\right)$ in $100 \mu \mathrm{l} 0.1 \%$ sodium citrate/ $0.1 \%$ Triton $\mathrm{X}-100$ containing the anti-8-OHdG antibody 15A3 (test sample, $2 \mu \mathrm{g} / \mathrm{ml}$ ) or a mouse IgG2a (isotype control, $2 \mu \mathrm{g} / \mathrm{ml})$. After washing twice with $1 \%$ NGS-PBS, spermatozoa were incubated in the dark ( $1 \mathrm{~h}$ at RT) with FITC-conjugated goat anti-mouse IgG (dilution 1:100 in $100 \mu$ l 1\% NGS-PBS). Then, samples were washed twice, re-suspended in $500 \mu \mathrm{l} \mathrm{PBS}$, stained with $7.5 \mu \mathrm{l} \mathrm{PI}(50 \mu \mathrm{g} / \mathrm{ml}$ in PBS) and incubated in the dark for 15 min at RT. An additional aliquot of sperm suspension was prepared with the same procedure used for the test sample but omitting the PI staining and used for instrumental compensation. In some experiments, the procedure of labelling was slightly modified as follows: i) $n=11$, the anti-8-OHdG antibody was pre-incubated with $8-\mathrm{OHdG}$ (at concentration $\sim 1000$-fold with respect to the antibody, $1 \mathrm{~h}$ at RT. Two commercially available nucleoside standards were tested); ii) $n=8$, before labelling procedure, spermatozoa were incubated with DTT, at $2 \mathrm{mM}$ for $45 \mathrm{~min}$ at $37^{\circ} \mathrm{C}$.

\section{Detection of OHdG by oxyDNA test}

Fixed spermatozoa were washed twice, permeabilized with $100 \mu \mathrm{l} 0.2 \%$ Triton-X (RT, for $15 \mathrm{~min}$ ) and split into two aliquots. After washing in wash solution (WS, $300 \mu \mathrm{l}$, provided by the manufacturer), the two aliquots were incubated $(1 \mathrm{~h}$ at $37^{\circ} \mathrm{C}$ ) in $100 \mu \mathrm{lWS}$ containing $3 \%$ BSA with (test sample) and without (negative control) the binding protein-FITC conjugate (BP-F, 1:200) provided by the manufacturer. At the end of incubation, spermatozoa were washed twice, re-suspended with PBS $(500 \mu \mathrm{l})$ and stained with PI. For fluorescence compensation, an additional aliquot was prepared as described 
earlier (incubation with the BP-F) except for the addition of PI. Preliminary experiments were performed in order to optimize the working concentration of BP-F. The BP-F was previously purified by incubation at room temperature for $1 \mathrm{~h}$ with about $1 \mathrm{mg}$ of activated charcoal powder by the same procedure described by others (De luliis et al. 2009, Aitken et al. 2010). By the same labelling procedure, we performed 11 competitive experiments where the BP-F was pre-incubated with $8-\mathrm{OHdG}$ (at concentration $\sim 1000$-fold respect to the BP-F, $1 \mathrm{~h}$ at RT). Two commercially available nucleoside standards were tested. In further competitive experiments, the procedure was slightly modified as follows: i) $n=3$, BSA was omitted in the incubation buffer containing the BP-F (with and without the excess of the nucleoside); ii) $n=4$, the BP-F was used before the purification step.

\section{8-OHdG detection by fluorescence microscopy}

Spermatozoa double stained for 8-OHdG and nuclei were laid on slides and green and red fluorescence were examined using a fluorescence microscope (Carl Zeiss, Axiolab A1 FL, Milan, Italy), equipped with Filter set 15 and 44 and an oil immersion $100 \times$ magnification objective.

\section{Flow cytometry}

Samples were acquired by a FACScan flow cytometer (Becton Dickinson, Mountain View, CA, USA) equipped with a $15 \mathrm{~mW}$ argon-ion laser for excitation. For each sample, 10000 events were recorded within the characteristic flame-shaped region in the forward scatter/side scatter (FSC/SSC) dot plot, which excludes debris and large cells (Muratori et al. 2010). Green fluorescence (FITC-conjugated goat anti-mouse IgG, BP-F) was revealed by an FL-1 (515-555 nm wavelength band) detector; red fluorescence of PI was detected by an FL-2 (563-607 nm wavelength band) detector.

Spermatozoa were analysed within the nucleated events (i.e. the events labelled with PI) of the FSC/SSC flame-shaped region (Muratori et al. 2010). The amount of labelled spermatozoa was scored as the percentage of spermatozoa having fluorescence intensities above a threshold excluding $\leq 1 \%$ of the events in the negative or isotype control. As PI staining reveals the occurrence of two different sperm populations (PI brighter, $\mathrm{PI}^{\text {bri }}$ and $\mathrm{PI}$ dimmer, $\mathrm{PI}^{\mathrm{dim}}$ ), the percentages of labelled spermatozoa were calculated in each of these populations and the two values were added (Muratori et al. 2008). In the experiments using the $80 \%$ and $40 / 80 \%$ fractions, the amounts of $8-\mathrm{OHdG}$ were calculated in the $\mathrm{Pl}^{\text {bri }}$ sperm population.

\section{Statistical analysis}

Results are expressed as mean \pm s.D. The distribution of each parameter was tested for normality, and non-normally distributed parameters were logarithmically transformed. Bivariate correlation was evaluated by calculating the Pearson's correlation coefficient $(r)$. ANOVA and the Student's $t$-test were used to assess statistically significant differences between the levels of spermatozoa with 8-OHdG: i) before and after hydroperoxide and DTT treatment; ii) determined using the
15A3 antibody or the BP-F of OxyDNA test and iii) in presence or not of high concentration of 8-OHdG during the incubation with BP-F or the antibody $15 \mathrm{~A} 3$. All statistical analyses were carried out using the SPSS version 17 Software for Windows (SPSS, Inc., Chicago, IL, USA).

\section{Declaration of interest}

The authors declare that there is no conflict of interest that could be perceived as prejudicing the impartiality of the research reported.

\section{Funding}

Supported by Regione Toscana (grant to G Forti), Ministry of Education and Scientific Research (PRIN 2009 project to E Baldi and FIRB project to S Marchiani).

\section{References}

Aitken RJ \& Curry BJ 2011 Redox regulation of human sperm function: from the physiological control of sperm capacitation to the etiology of infertility and DNA damage in the germ line. Antioxidants \& Redox Signaling 14 367-381. (doi:10.1089/ars.2010.3186)

Aitken RJ, Harkiss D \& Buckingham DW 1993 Analysis of lipid peroxidation mechanisms in human spermatozoa. Molecular Reproduction and Development 35 302-315. (doi:10.1002/mrd.1080350313)

Aitken RJ, De Iuliis GN, Finnie JM, Hedges A \& McLachlan RI 2010 Analysis of the relationships between oxidative stress, DNA damage and sperm vitality in a patient population: development of diagnostic criteria. Human Reproduction 25 2415-2426. (doi:10.1093/humrep/deq214)

Athayde KS, Cocuzza M, Agarwal A, Krajcir N, Lucon AM, Srougi M \& Hallak J 2007 Development of normal reference values for seminal reactive oxygen species and their correlation with leukocytes and semen parameters in a fertile population. Journal of Andrology 28 613-620. (doi:10.2164/jandrol.106.001966)

Badouard C, Ménézo Y, Panteix G, Ravanat JL, Douki T, Cadet J \& Favier A 2008 Determination of new types of DNA lesions in human sperm. Zygote 16 9-13. (doi:10.1017/S0967199407004340)

Banihani S, Sharma R, Bayachou M, Sabanegh E \& Agarwal A 2012 Human sperm DNA oxidation, motility and viability in the presence of L-carnitine during in vitro incubation and centrifugation. Andrologia 44 (Suppl) 505-512. (doi:10.1111/j.1439-0272.2011.01216.x)

Bellver J, Meseguer M, Muriel L, García-Herrero S, Barreto MA, Garda AL, Remohí J, Pellicer A \& Garrido N 2010 Y chromosome microdeletions, sperm DNA fragmentation and sperm oxidative stress as causes of recurrent spontaneous abortion of unknown etiology. Human Reproduction 25 1713-1721. (doi:10.1093/humrep/deq098)

Chen H, Zhao HX, Huang XF, Chen GW, Yang ZX, Sun WJ, Tao MH, Yuan Y, Wu JQ, Sun F et al. 2012 Does high load of oxidants in human semen contribute to male factor infertility? Antioxidants \& Redox Signaling 16 754-759. (doi:10.1089/ars.2011.4461)

Cocuzza M, Sikka SC, Athayde KS \& Agarwal A 2007 Clinical relevance of oxidative stress and sperm chromatin damage in male infertility: an evidence based analysis. International Brazilian Journal of Urology 33 603-621. (doi:10.1590/S1677-55382007000500002)

Cohen-Bacrie P, Belloc S, Ménézo YJ, Clement P, Hamidi J \& Benkhalifa M 2009 Correlation between DNA damage and sperm parameters: a prospective study of 1,633 patients. Fertility and Sterility 91 1801-1805. (doi:10.1016/j.fertnstert.2008.01.086)

Conners R, Hooley E, Clarke AR, Thomas S \& Brady RL 2006 Recognition of oxidatively modified bases within the biotin-binding site of avidin. Journal of Molecular Biology 357 263-274. (doi:10.1016/ j.jmb.2005.12.054)

De luliis GN, Thomson LK, Mitchell LA, Finnie JM, Koppers AJ, Hedges A, Nixon B \& Aitken RJ 2009 DNA damage in human spermatozoa is highly 
correlated with the efficiency of chromatin remodeling and the formation of 8-hydroxy-2'-deoxyguanosine, a marker of oxidative stress. Biology of Reproduction 81 517-524. (doi:10.1095/biolreprod.109.076836)

Evenson DP, Jost LK, Marshall D, Zinaman MJ, Clegg E, Purvis K, de Angelis P \& Claussen OP 1999 Utility of the sperm chromatin structure assay as a diagnostic and prognostic tool in the human fertility clinic. Human Reproduction 14 1039-1049. (doi:10.1093/humrep/14.4.1039)

Gomez E, Buckingham DW, Brindle J, Lanzafame F, Irvine DS \& Aitken RJ 1996 Development of an image analysis system to monitor the retention of residual cytoplasm by human spermatozoa: correlation with biochemical markers of the cytoplasmic space, oxidative stress, and sperm function. Journal of Andrology 17 276-287.

Kao SH, Chao HT, Chen HW, Hwang TI, Liao TL \& Wei YH 2008 Increase of oxidative stress in human sperm with lower motility. Fertility and Sterility 89 1183-1190. (doi:10.1016/j.fertnstert.2007.05.029)

Lanzafame FM, La Vignera S, Vicari E \& Calogero AE 2009 Oxidative stress and medical antioxidant treatment in male infertility. Reproductive BioMedicine Online 19 638-659. (doi:10.1016/j.rbmo.2009.09.014)

Lewis SE 2007 Is sperm evaluation useful in predicting human fertility? Reproduction 134 31-40. (doi:10.1530/REP-07-0152)

Li K, Shang X \& Chen Y 2004 High-performance liquid chromatographic detection of lipid peroxidation in human seminal plasma and its application to male infertility. Clinica Chimica Acta 346 199-203. (doi:10.1016/j.cccn.2004.03.013)

Marchiani S, Tamburrino L, Maoggi A, Vannelli GB, Forti G, Baldi E \& Muratori M 2007 Characterization of M450 bodies in human semen; evidence that they are apoptotic bodies. Molecular Human Reproduction 13 621-631. (doi:10.1093/molehr/gam046)

Marchiani S, Tamburrino L, Giuliano L, Nosi D, Sarli V, Gandini L, Piomboni P, Belmonte G, Forti G, Baldi E et al. 2011 Sumo1-ylation of human spermatozoa and its relationship with semen quality. International Journal of Andrology 34 581-593. (doi:10.1111/j.13652605.2010.01118.x)

Meamar M, Zribi N, Cambi M, Tamburrino L, Marchiani S, Filimberti E, Fino MG, Biggeri A, Menezo Y, Forti G et al. 2012 Sperm DNA fragmentation induced by cryopreservation: new insights and effect of a natural extract from Opuntia ficus-indica. Fertility and Sterility $\mathbf{9 8}$ 326-333. (doi:10.1016/j.fertnstert.2012.05.001)

Meseguer M, Martínez-Conejero JA, O'Connor JE, Pellicer A, Remohí J \& Garrido N 2008 The significance of sperm DNA oxidation in embryo development and reproductive outcome in an oocyte donation program: a new model to study a male infertility prognostic factor. Fertility and Sterility 89 1191-1199. (doi:10.1016/j.fertnstert.2007.05.005)

Montjean D, Ménézo Y, Benkhalifa M, Cohen M, Belloc S, Cohen-Bacrie P \& De Mouzon J 2010 Malonaldehyde formation and DNA fragmentation: two independent sperm decays linked to reactive oxygen species. Zygote 18 265-268. (doi:10.1017/S0967199409990311)

Morpurgo M, Radu A, Bayer EA \& Wilchek M 2004 DNA condensation by high-affinity interaction with avidin. Journal of Molecular Recognition 17 558-566. (doi:10.1002/jmr.689)

Muratori M, Maggi M, Spinelli S, Filimberti E, Forti G \& Baldi E 2003 Spontaneous DNA fragmentation in swim-up selected human spermatozoa during long term incubation. Journal of Andrology 24 253-262.

Muratori M, Marchiani S, Maggi M, Forti G \& Baldi E 2006 Origin and biological significance of DNA fragmentation in human spermatozoa. Frontiers in Bioscience 11 1491-1499. (doi:10.2741/1898)

Muratori M, Marchiani S, Tamburrino L, Tocci V, Failli P, Forti G \& Baldi E 2008 Nuclear staining identifies two populations of human sperm with different DNA fragmentation extent and relationship with semen parameters. Human Reproduction 23 1035-1043. (doi:10.1093/humrep/ den058)

Muratori M, Tamburrino L, Tocci V, Costantino A, Marchiani S, Giachini C, Laface I, Krausz C, Meriggiola MC, Forti G et al. 2010 Small variations in crucial steps of TUNEL assay coupled to flow cytometry greatly affect measures of sperm DNA fragmentation. Journal of Andrology 31 336-345. (doi:10.2164/jandrol.109.008508)

Ni ZY, Liu YQ, Shen HM, Chia SE \& Ong CN 1997 Does the increase of 8-hydroxydeoxyguanosine lead to poor sperm quality? Mutation Research 381 77-82. (doi:10.1016/S0027-5107(97)00151-6)

Pasqualotto FF, Sharma RK, Kobayashi H, Nelson DR, Thomas AJ Jr \& Agarwal A 2001 Oxidative stress in normospermic men undergoing infertility evaluation. Journal of Andrology 22 316-322.

Pasqualotto FF, Sundaram A, Sharma RK, Borges E Jr, Pasqualotto EB \& Agarwal A 2008 Semen quality and oxidative stress scores in fertile and infertile patients with varicocele. Fertility and Sterility 89 602-607. (doi:10.1016/j.fertnstert.2007.03.057)

Sakkas D \& Alvarez JG 2010 Sperm DNA fragmentation: mechanisms of origin, impact on reproductive outcome, and analysis. Fertility and Sterility 93 1027-1036. (doi:10.1016/j.fertnstert.2009.10.046)

Santiso R, Tamayo M, Gosálvez J, Meseguer M, Garrido N \& Fernández JL 2010 Simultaneous determination in situ of DNA fragmentation and 8-oxoguanine in human sperm. Fertility and Sterility 93 314-318. (doi:10.1016/j.fertnstert.2009.07.969)

Shen HM, Chia SE \& Ong CN 1999 Evaluation of oxidative DNA damage in human sperm and its association with male infertility. Journal of Andrology 20 718-723.

Struthers L, Patel R, Clark J \& Thomas S 1998 Direct detection of 8-oxodeoxyguanosine and 8-oxoguanine by avidin and its analogues. Analytical Biochemistry 255 20-31. (doi:10.1006/abio.1997.2354)

Takakura Y, Oka N, Kajiwara H \& Tsunashima M 2012 Engineering of novel tamavidin 2 muteins with lowered isoelectric points and lowered nonspecific binding properties. Journal of Bioscience and Bioengineering 114 485-489. (doi:10.1016/j.jbiosc.2012.06.009)

Tamburrino L, Marchiani S, Montoya M, Elia Marino F, Natali I, Cambi M, Forti G, Baldi E \& Muratori M 2012 Mechanisms and clinical correlates of sperm DNA damage. Asian Journal of Andrology 14 24-31. (doi:10.1038/aja.2011.59)

Thomson LK, Fleming SD, Aitken RJ, De luliis GN, Zieschang JA \& Clark AM 2009 Cryopreservation-induced human sperm DNA damage is predominantly mediated by oxidative stress rather than apoptosis. Human Reproduction 24 2061-2070. (doi:10.1093/humrep/dep214)

Tremellen K 2008 Oxidative stress and male infertility - a clinical perspective. Human Reproduction Update 14 243-258. (doi:10.1093/ humupd/dmn004)

Twigg JP, Fulton N \& Gomez E 1988 Analysis of the impact of intracellular reactive oxygen species generation on the structural and functional integrity of human spermatozoa: lipid peroxidation, DNA fragmentation and effectiveness of antioxidant. Human Reproduction 13 1429-1436. (doi:10.1093/humrep/13.6.1429)

World Health Organization 1999. In WHO Laboratory Manual for the Examination of Human Semen and Sperm-Cervical Mucus Interactions, 4th edn. Cambridge, UK: Cambridge University Press.

Zribi N, Feki Chakroun N, El Euch H, Gargouri J, Bahloul A \& Ammar Keskes L 2010 Effects of cryopreservation on human sperm deoxyribonucleic acid integrity. Fertility and Sterility 93 159-166. (doi:10.1016/ j.fertnstert.2008.09.038)

Zribi N, Chakroun NF, Elleuch H, Abdallah FB, Ben Hamida AS, Gargouri J, Fakhfakh F \& Keskes LA 2011 Sperm DNA fragmentation and oxidation are independent of malondialdheyde. Reproductive Biology and Endocrinology 9 47. (doi:10.1186/1477-7827-9-47)

Received 11 October 2012

First decision 5 November 2012

Revised manuscript received 17 December 2012

Accepted 7 January 2013 